\title{
IN THIS ISSUE
}

This special issue responds to publications arising from presentations at the 2020 CPCE Health Conference held in Hong Kong in January. The conference was conducted by the College of Professional and Continuing Education of the Hong Kong Polytechnic University in association with a number a number of organisations including the Centre for Ageing and Healthcare Management Research and our colleagues from the Hong Kong College of Health Service Executives.

The theme of the conference was 'Ageing with Health and Dignity - Implications for Public Policy, Service Delivery Workforce, Technology and Financing'.

A further general call was made by APJHM for this themed issue and we were pleased to accept an appropriate article from health management academic colleagues from Iran.

The editorial for this issue is appropriately provided by the Conference Chair, Professor Peter Yuen who is the Dean of CPCE at the Hong Kong Polytechnic University. The first article in this issue is provided by Fung and Fong from the School of Professional Development of the Hong Kong Polytechnic University. In a case study it describes 'service learning' as a useful learning activity for students to understand and analyse the health and social conditions of the elderly in a practical environment. The experience is described as 'Treasure in elderly care learning'.

In a similar context the second research article is provided by YLee, Fong, $\mathrm{Ng}$ and Chow. It is described as a community service-based learning initiative conducted in four selected elderly centres in Hong Kong. The students identified and improved skills and learned better ways to communicate with elders and to respond quickly to changes in designed activities to elders. In the next article $\mathrm{Ho}$ and $\mathrm{Ng}$ describe the 'improvement of the elderly health voucher scheme in Hong Kong. The scheme provides financial support by the government to the elderly for having more choices in selecting private primary health care services. The scheme is described as having a high participation rate, yet the amount of the subsidy is not sufficient to meets the needs of the elderly. The study suggests a need for increased funding and improved standards of monitoring the performance of service providers.

In our fourth article $\mathrm{Ng}$, Yim and Fong describe a system to reduce the burden of the ageing population through 'time banking'. This is described as an approach to maximise social capital through exchanging one's time to serve others that can be saved as a credit to use later when requiring services. The review article suggests that further time is required for governmental, technical, and educational support. In our next article Tavitiyaman, from the Hong Kong Polytechnic University and Saiprasert from the Chiang Mai University Thailand. This research describes the medical quality and wellbeing perceptions of senior tourists in their quest for healthcare in other countries.

Yee, Fong and $\mathrm{Ng}$ in our next article provide a descriptive overview of palliative care services in Hong Kong. They then suggest that guidelines are required with criteria to screen patients for palliative care needs in advance. They suggest that professional education and training be expanded as well as the need to improve public knowledge and education. They also suggest increased funding on palliative care as well as the need for further research.

The next two articles relate to the concept of 'green burials.' The first article by Lau, Yee, $\mathrm{Ng}$ and Fong describes the context of decreasing space and capacity for traditional funerals much favoured in Chinese cultural contexts and a lack of readiness to adopt the concept of green burials. They provide the context and challenges in attempts to promote more and better use of this concept. 
The second of these two articles Lau, Tang, Chan, Ng and Leung in an approach that is attempting to utilise the deployment of virtual reality to promote the concept of 'green burial.' The authors ask the questions about the potential of this technology to debunk current community perceptions and change the community acceptance of this practice.

In our next article health management colleagues from Iran present an article on 'active ageing management: designing a model'. The article by Gholipour, Abadi Farahani, Riahi and Hajinabi suggest that active ageing practices have not received sufficient attention in Iran. Their research seeks to identify factors that might substantiate a policy model to address and improve public policy around active ageing.

Lam, Ng and Yee provide our next article on the improvement of safety in operating theatres by training and teamwork. They suggest a management plan that focusses on human resources, professional training and retention of staff and teamwork. Lai and Kaur from the Hong Kong College of Community Health Practitioners next provide a timely article on home hygiene and the prevention of infections reminding the readers that proper practices lead to the reduction of infectious diseases. Yip and Chong provide an explanatory study on the role and responsibility of clinical pharmacists in a rheumatology clinic. Arthritis is a leading chronic disorder amongst older people. The research aim was to improve patient medication adherence and enhance medication safety. Kwong and Fong continue this theme in the next article on the quality management of inpatient medical administration in Hong Kong public hospitals. They suggest that the elimination of potential risks arising from prescribing, dispensing and drug administration processes brings the achievement of medication safety. Wong and Chong provide our final research article providing a retrospective evaluation on patient screening and counselling service on direct-acting antivirals against hepatitis $C$.

The APJHM appreciates the contributions of our authors and of the organisers of the conference in the submission and acceptance of these articles.

DS Briggs AM

Chief Editor 